\title{
Intercostal artery pseudoaneurysm following thoracentesis: multi-modal imaging and treatment
}

\author{
Kaitlyn P. Casper ${ }^{1}$, Paul J. Sanchirico ${ }^{2}$ and David C. Pfeiffer ${ }^{3 *}$ (D)
}

\begin{abstract}
Background: A pseudoaneurysm occurs as the result of a contained rupture of an arterial wall, yielding a perfused sac that communicates with the arterial lumen. Pseudoaneurysm of an intercostal artery is an extremely rare event but it carries with it a significant risk of rupture and subsequent hemothorax. It must be considered as a potential complication of thoracentesis.

Case presentation: Here, we report a rare case of an intercostal artery pseudoaneurysm following thoracentesis in an 82-year old male. The patient presented with respiratory distress 1 day after a therapeutic thoracentesis had been performed. Computed tomography $(\mathrm{CT})$ with contrast revealed a left intercostal pseudoaneurysm with hemothorax and adjacent compressive atelectasis. Doppler ultrasound revealed bidirectional blood flow in the pseudoaneurysm sac. An intercostal arteriogram and thoracic aortogram aided in confirmation of the pseudoaneurysm and successful treatment with coil embolization.

Conclusions: An intercostal pseudoaneurysm complication following thoracentesis is very rare but important to rule out as a possible cause of hemothorax after the procedure. Capturing this finding with the aid of multiple imaging modalities allowed for diagnostic certainty and rapid treatment with coil embolization, leading to a successful patient recovery.
\end{abstract}

Keywords: Pseudoaneurysm, Thoracentesis, Intercostal artery, Hemothorax, Coil embolism, Multi-modal imaging

\section{Background}

Thoracentesis is a commonly performed procedure in the diagnosis and possible treatment of pleural effusion. Typical complications of the procedure include pneumothorax, bleeding including hemothorax, and re-expansion pulmonary edema [1]. A pseudoaneurysm results from damage to an arterial wall in a manner that permits blood to dissect into tissues of the vessel wall, forming a perfused sac that communicates with the arterial lumen [2,3]. Pseudoaneurysm of an intercostal artery is an extremely rare condition, with relatively few documented cases in the literature. Previous reports have documented it as a complication of chest trauma or surgical procedures [4-13]. It carries with it a significant risk of rupture and subsequent hemothorax and therefore prompt and accurate

\footnotetext{
* Correspondence: dpfeiffer@uidaho.edu

${ }^{3}$ WWAMI Medical Education Program and Department of Biological Sciences, University of Idaho, 875 Perimeter Drive, Moscow, ID 83844-3051, USA Full list of author information is available at the end of the article
}

identification is important. We describe a case of an intercostal pseudoaneurysm complication of a thoracentesis that was documented on multiple imaging modalities and successfully treated with coil embolization.

\section{Case presentation}

An 82-year-old male was admitted to the emergency department for worsening shortness of breath and hypoxia. He was admitted a week after he was diagnosed with a left ninth rib fracture secondary to a fall. He had long-standing history of chronic obstructive pulmonary disease, coronary artery disease, and peripheral vascular disease. Chest radiographs revealed a left pleural effusion and possible infiltrate. The patient was initially treated with a nebulizer, prednisone, and empiric antibiotic coverage with ceftriaxone and azithromycin. The patient failed to improve with the medical interventions and a therapeutic thoracentesis was 
performed. The thoracentesis was completed with ultrasound guidance, with the puncture made above the 11th rib at mid chest on the left. The pleural effusion was found to be frank blood. No immediate complications were noted, and the patient was taken to recovery. The next day the patient was found to be in respiratory distress. A chest x-ray revealed an opaque left hemithorax that was likely rapid accumulation of pleural fluid (Fig. 1). A follow-up contrast-enhanced computed tomography (CT) of the chest performed during the arterial phase revealed a left intercostal pseudoaneurysm with hemothorax and adjacent compressive atelectasis (Fig. 2). Ultrasound of the left chest wall was performed (Fig. 3) directly over the thoracentesis site and doppler flow revealed bidirectional fluid flow, indicating the presence of a large pseudoaneurysm (Fig. 4).

Following identification of the left intercostal pseudoaneurysm, the patient underwent a thoracic aortogram and multiple-level left intercostal angiogram (Fig. 5) under IV conscious sedation. Selective catheterization of the T5, T6, and T7 intercostal arteries was unsuccessful in identifying the pseudoaneurysm. Selective catheterization of T10 and T11 intercostal arteries was performed with a C2 Cobra catheter, following multiple catheter exchanges due to the patient's atherosclerotic vessels. The pseudoaneurysm was ultimately found to have a left T10 origin and the $\mathrm{C} 2$ Cobra catheter was exchanged for a microcatheter. Once access was gained, coil embolization of the pseudoaneurysm was performed with a series of 15 Axium micro coils. Significant room was left on both sides of the pseudoaneurysm and a follow-up angiogram was performed via the microcatheter, then a 5-French Cobra catheter. The follow-up angiogram demonstrated no further filling of the pseudoaneurysm (Fig. 6). The catheter was removed and a Perclose device was placed in the left groin for hemostasis. Following completion of the procedure, the patient was taken to recovery. The patient proceeded to return to his baseline following medical management during the remainder of his hospital stay and was discharged home after 5 days.

\section{Discussion}

Thoracentesis is a very common procedure used to remove pleural fluid for diagnostic or therapeutic purposes. The most common complications of the procedure include pneumothorax, bleeding includinghemothorax, and re-expansion pulmonary edema [1]. Following the introduction of ultrasound guiding thoracentesis, the complication rates have significantly decreased due to improved accuracy of needle placement [14]. As in our case, hemothorax is to be considered if

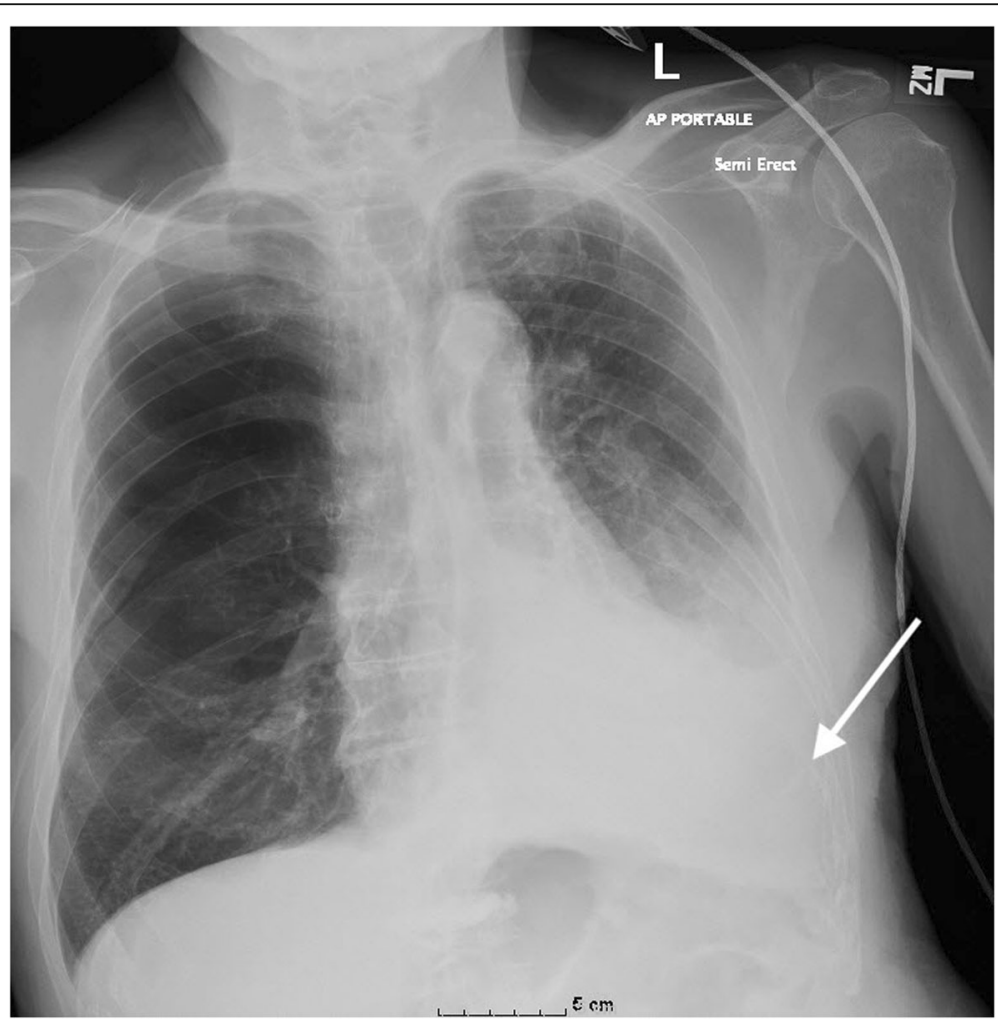

Fig. 1 AP (anteroposterior) chest plain radiograph in an 82-year old male, following thoracentesis. Note the significant pleural effusion with compressive atelectasis (arrow) in the left lower hemithorax 


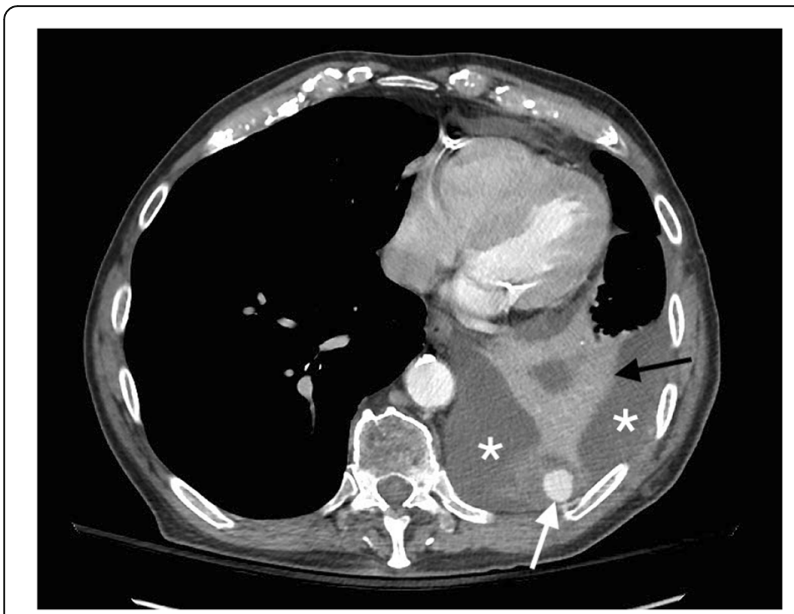

Fig. 2 IV contrast-enhanced computed tomography (CT) image, axial projection. The left intercostal artery pseudoaneurysm (white arrow) with hemothorax (asterisks) and adjacent compression atelectasis (black arrow) can be seen the patient has a rapid accumulation of pleural fluid or signs of respiratory distress.

An intercostal pseudoaneurysm is an extremely rare phenomenon, seldomly described in literature. Etiology of previously described intercostal artery pseudoaneurysms includes blunt thoracic trauma $[7,8,12]$, and surgical procedures including thoracoscopic sympathectomy [4], percutaneous biliary procedure [5], laparoscopic procedure [6], sternotomy [9], aortic valve implantation [11], and biopsy $[10,13]$. To the best of our knowledge, only one other case reported this complication as the result of thoracentesis; however, in that case the patient was unable to undergo successful embolization of the pseudoaneurysm [15].

Our patient's intercostal pseudoaneurysm was captured with the aid of three different imaging modalities prior to treatment intervention. The pseudoaneurysm was initially detected on the contrast-enhanced CT. The procedure was performed during arterial phase and this played a pivotal role in the diagnostic process. Doppler ultrasound identifying the possible pseudoaneurysm revealed a "yin-yang sign" which can be characteristic of the complication. This radiological sign has been described to represent the bidirectional flow of blood in

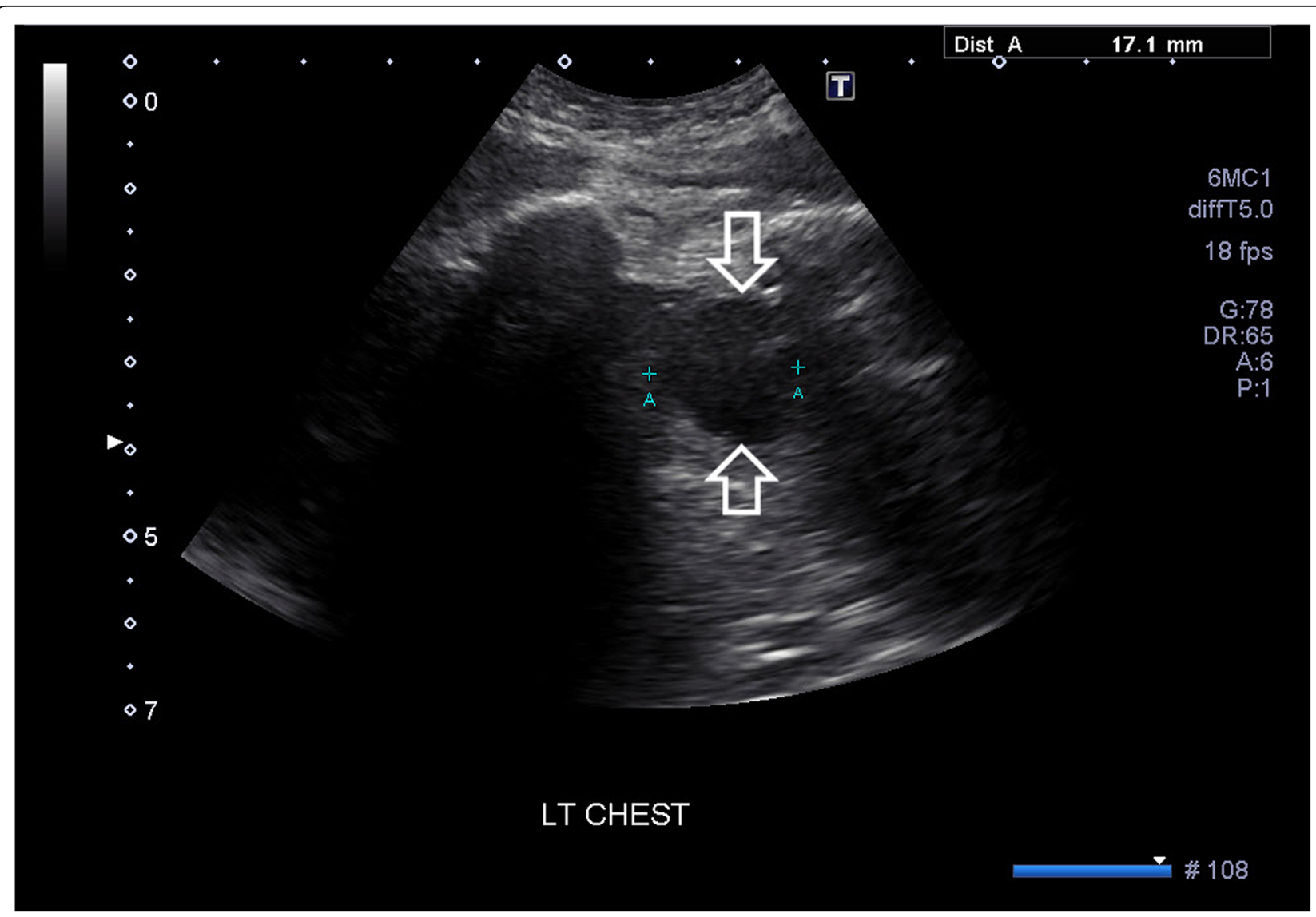

Fig. 3 Grey scale ultrasound image of the left intercostal artery pseudoaneurysm. Arrows delineate the pseudoaneurysm 


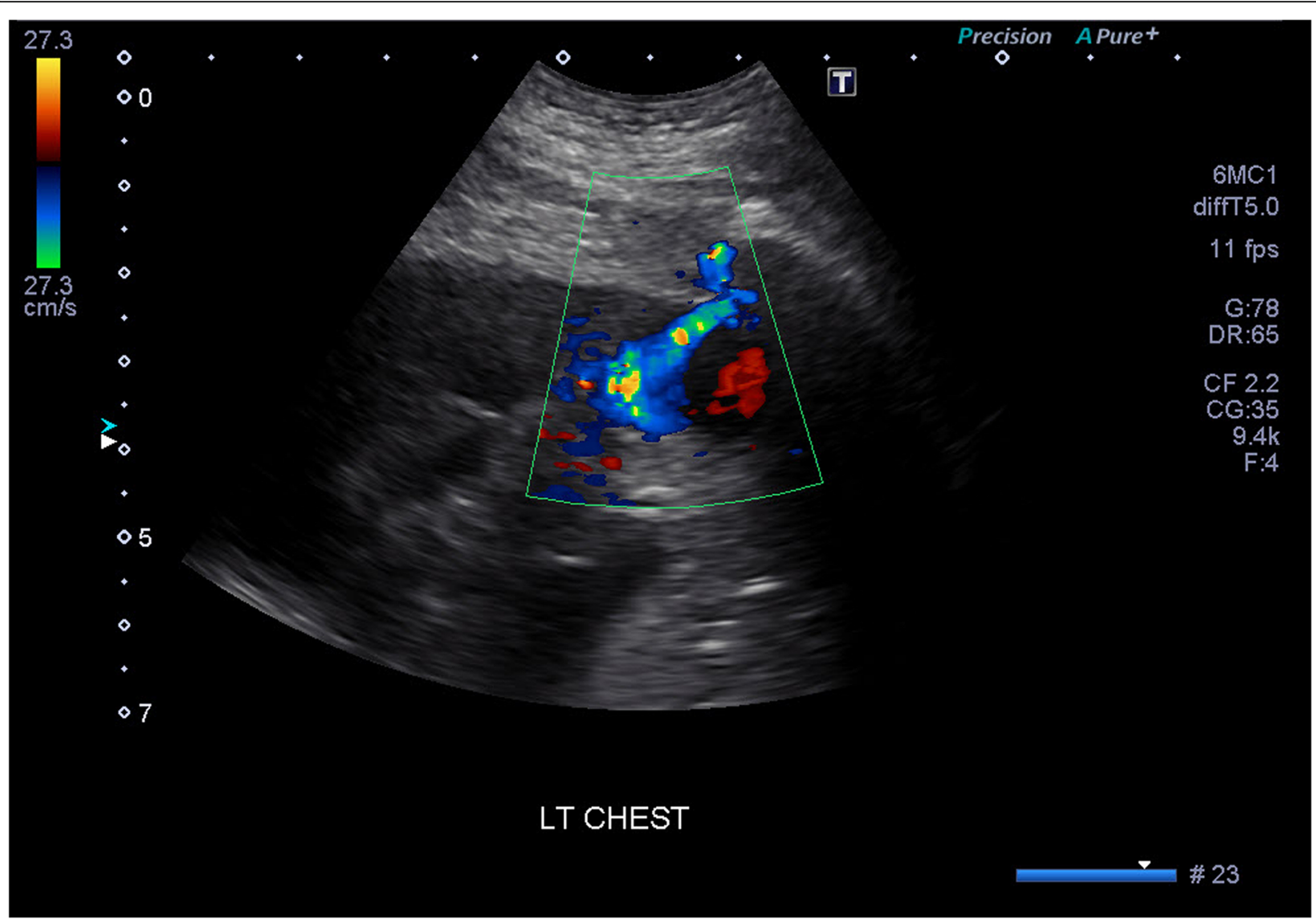

Fig. 4 Color Doppler ultrasound image of the intercostal artery pseudoaneurysm. Note the characteristic yin-yang (red-blue) blood flow present in the pseudoaneurysm

the pseudoaneurysm sac and is helpful in the diagnostic confirmation $[8,13]$.

The gold standard for diagnosing pseudoaneurysms on imaging is conventional angiography, however, less invasive modalities are increasing in use including ultrasonography, computed tomographic angiography (CTA), and magnetic resonance angiography (MRA) [8]. CTA and MRA both have been demonstrated to be reliable, high-quality imaging tools for the evaluation of vascular abnormalities in general and provide the advantages of rapid examination and short
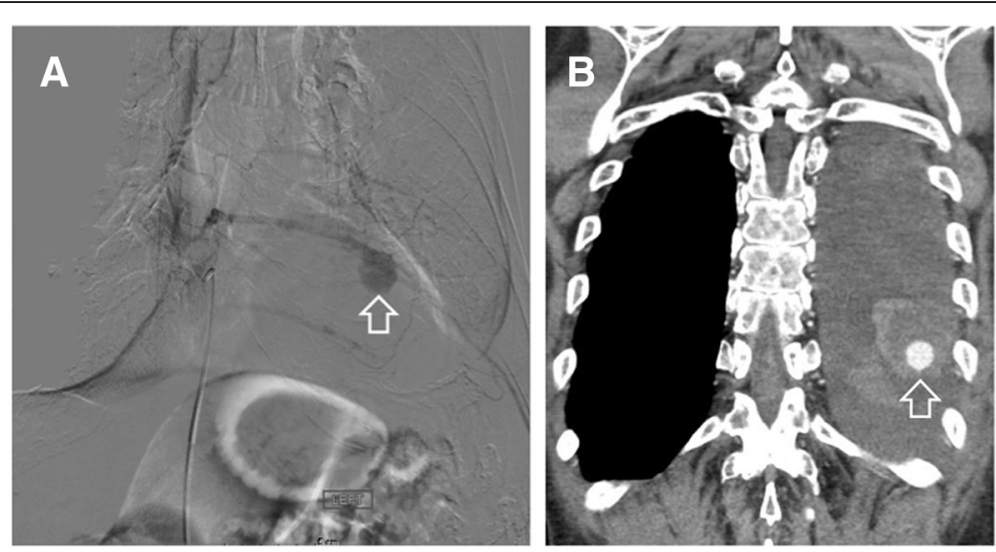

Fig. 5 a Selective digital subtraction angiogram of the intercostal artery pseudoaneurysm (arrow). b IV contrast-enhanced computed tomography (CT) image, coronal projection, depicting the pseudoaneurysm (arrow) 


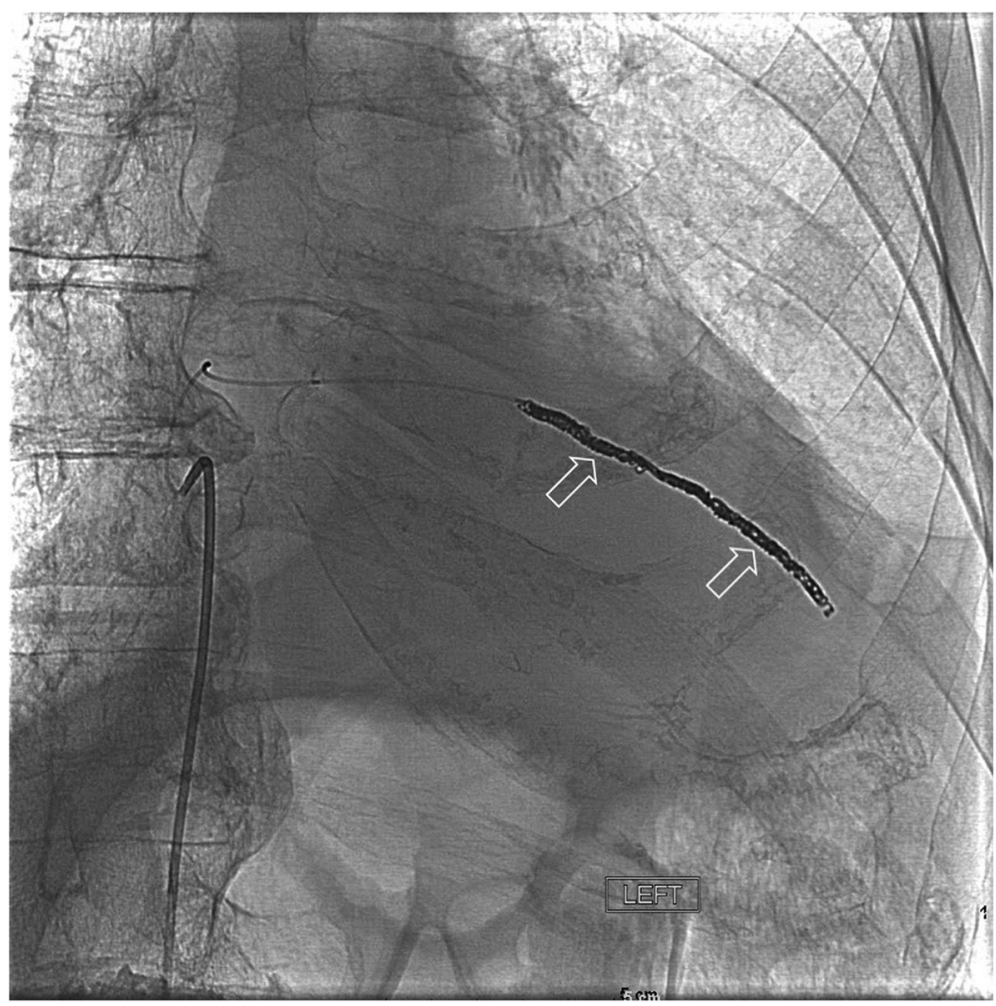

Fig. 6 Digital subtraction angiogram image demonstrating successful occlusion of the intercostal artery pseudoaneurysm by means of coil embolization. The coils are clearly visible (arrows)

scanning times [17-19]. Disadvantages associated with CTA include patient exposure to ionizing radiation [20, 21]. Contrast-enhanced MRA carries with it the risk of adverse reactions to gadolinium-based contrast material, a serious concern in patients with poor renal function. CT techniques that use less contrast agent could provide viable alternatives, although more studies on their effectiveness in vascular imaging are needed [19]. Newer contrast-enhanced MRA methods that utilize dose reduction at $3.0 \mathrm{~T}$, novel contrast agents, parallel imaging methods, and time-resolved imaging for contrast kinetics have been shown to be effective in the evaluation of other vascular disorders [22-24] and may have potential in the diagnosis of pseudoaneurysms.

Early treatment of the pseudoaneurysm before rupture is critical in the success of the patient's recovery. If rupture did occur, the patient would be at risk of a possible life-threatening hemorrhage [8]. Currently, coil embolization is the most common treatment of intercostal artery pseudoaneurysms, followed by covered stent placement $[8,16]$. In previously reported cases, the return of blood flow has been documented following embolization. These cases required re-treatment with thrombin, $n$-butyl cyanoacrylate or re-embolization at a different location $[8$, 16]. Our patient had successful primary embolization with Axium coils and a follow up angiogram revealed no further filling of the aneurysm.

\section{Conclusion}

An intercostal artery pseudoaneurysm as a complication of thoracentesis is an extremely unusual condition but it is important to rule out as a possible cause of hemothorax after the procedure. Capturing this finding with the aid of multiple imaging modalities allowed for diagnostic certainty and rapid treatment with coil embolization, leading to a successful patient recovery.

\section{Abbreviation}

CT: computed tomography

\section{Acknowledgements}

Not applicable.

\section{Funding}

Not applicable.

\section{Availability of data and materials}

Not applicable.

\section{Authors' contributions}

KPC, PJS, and DCP all made substantial contributions to conception and design of the manuscript. Each has been involved in drafting the manuscript and its critical revision. Each has read and given final approval of the version to be published and each has participated sufficiently in the work to take public responsibility for appropriate portions of the content. All have agreed to be accountable for all aspects of the work in ensuring that questions 
related to the accuracy or integrity of any part of the work are appropriately investigated and resolved. All authors read and approved the final manuscript.

\section{Authors' information}

KPC is a medical student in the WWAMI Medical Education Program at the University of Washington School of Medicine, 1959 NE Pacific St, Seattle, WA, 98195. PJS is a diagnostic radiologist and vascular and interventional radiologist at St Joseph Regional Medical Center, 415 6th St, Lewiston, ID, 83501. DCP is a professor in the Department of Biological Sciences and the WWAMI Medical Education Program at the University of Idaho, 875 Perimeter Drive, Moscow, ID, 83844-3051, USA

\section{Ethics approval and consent to participate}

Not applicable.

\section{Consent for publication}

The patient has provided written informed consent to the clinical details including images of the case being submitted and published as a case report.

\section{Competing interests}

The authors declare they have no competing interests.

\section{Publisher's Note}

Springer Nature remains neutral with regard to jurisdictional claims in published maps and institutional affiliations.

\section{Author details}

'WWAMI Medical Education Program, University of Washington School of Medicine, 1959 NE Pacific St, Seattle, WA 98195, USA. ² St Joseph Regional Medical Center, 415 6th St, Lewiston, ID 83501, USA. ${ }^{3}$ WWAMI Medical Education Program and Department of Biological Sciences, University of Idaho, 875 Perimeter Drive, Moscow, ID 83844-3051, USA

Received: 28 December 2018 Accepted: 12 April 2019 Published online: 27 April 2019

\section{References}

1. Cantey EP, Walter JM, Corbridge T, Barsuk JH. Complications of thoracentesis: incidence, risk factors, and strategies for prevention. Curr Opin Pulm Med. 2016;22:378-85. https://doi.org/10.1097/MCP.0000000000000285.

2. Schwartz LB, Clark ET, Gewertz BL. Anastomotic and other pseudoaneurysms. In: Rutherford RB, editor. Vascular surgery. 5th ed. Philadelphia: Saunders; 2000. p. 752-63

3. Saad NEA, Saad WEA, Davies MG, Waldman DL, Fultz PJ, Rubens DJ. Pseudoaneurysms and the role of minimally invasive techniques in their management. RadioGraphics. 2005;25:S173-89. https://doi.org/10.1148/rg. 25 si055503.

4. Atherton WG, Morgan WE. False aneurysm of an intercostal artery after thoracoscopic sympathectomy. Ann R Coll Surg Engl. 1997;79:229-30.

5. Casas JD, Perendreu J, Gallart A, Muchart J. Intercostal artery pseudoaneurysm after a percutaneous biliary procedure: diagnosis with $\mathrm{CT}$ and treatment with transarterial embolization. J Comput Assist Tomogr. 1997:21:729-30.

6. Bluebond-Langner R, Pinto P, Kim FJ, Hsu T, Jarrett TW. Recurrent bleeding from intercostal arterial pseudoaneurysm after retroperitoneal laparoscopic radical nephrectomy. Urology. 2002;60:1111. https://doi.org/10.1016/S00904295(02)01998-2.

7. Aoki T, Okada A, Tsuchida M, Hayashi J. Ruptured intercostal artery pseudoaneurysm after blunt thoracic trauma. Thorac Cardiovasc Surg. 2003; 51:346-7. https://doi.org/10.1055/s-2003-45512.

8. Sekino S, Takagi H, Kubota H, Kato T, Matsuno Y, Umemoto T. Intercostal artery pseudoaneurysm due to stab wound. J Vasc Surg. 2005;42:352-6. https://doi.org/10.1016/J.JVS.2005.03.058.

9. Fernandez Alonso S, Azcona CM, Heredero AF, de Cubas LR. Post-sternotomy intercostal artery pseudoaneurysm. Sonographic diagnosis and thrombosis by ultrasound-guided percutaneous thrombin injection. Interact Cardiovasc Thorac Surg. 2009;9:722-4. https://doi.org/10.1510/icvts.2009.208116.

10. Melloni G, Bandiera A, Crespi G, Zannini P. Intercostal artery pseudoaneurysm after computed tomography-guided percutaneous fine needle aspiration lung biopsy. J Thorac Imaging. 2012;27:W48-9. https://doi org/10.1097/RTI.0b013e3182107430.

11. Lenders G, Van Schil P, Rodrigus I, Bosmans J. Intercostal artery pseudoaneurysm: a rare complication of transaortic transcatheter aortic valve implantation. Interact Cardiovasc Thorac Surg. 2012;15:550-2. https://doi.org/10.1093/icvts/ivs188.

12. Gutierrez Romero DF, Barrufet M, Lopez-Rueda A, Burrel M. Ruptured intercostal artery pseudoaneurysm in a patient with blunt thoracic trauma: diagnosis and management. BMJ Case Rep. 2014. https://doi.org/10.1136/ bcr-2013-202019.

13. Vajtai Z, Roy N. Intercostal artery pseudoaneurysm after ultrasound-guided liver biopsy: a case report and review of the literature. Ultrasound Q. 2015 31:63-5. https://doi.org/10.1097/RUQ.0000000000000074

14. Mercaldi MPHCJ, Lanes SF. Ultrasound guidance decreases complications and improves the cost of care among patients undergoing thoracentesis and paracentesis. Chest. 2013;143:532-8. https://doi.org/10.1378/chest.12-0447.

15. Long SS, Johnson PT, Fishman EK. Intercostal artery pseudoaneurysm due to thoracentesis: diagnosis with three-dimensional computed tomographic angiography. J Comput Assist Tomogr. 2012;36:100-2. https://doi.org/10. 1097/RCT.0b013e318243250a.

16. Yamakado K, Nakatsuka A, Tanaka N, Takano K, Matsumura K, Takeda K. Transcatheter arterial embolization of ruptured pseudoaneurysms with coils and n-butyl cyanoacrylate. J Vasc Interv Radiol. 2000;11:66-72.

17. Met R, Bipat S, Legemate DA, Reekers JA, Koelemay MJ. Diagnostic performance of computed tomography angiography in peripheral arterial disease: a systematic review and meta-analysis. JAMA. 2009;301:415-24. https://doi.org/10.1001/jama.301.4.415.

18. Hartung MP, Grist TM, François CJ. Magnetic resonance angiography: current status and future directions. J Cardiovasc Magn Reson. 2011;13:1929. https://doi.org/10.1186/1532-429X-13-19.

19. Razek AAK, Denewer AT, Hegazy MAF, Hafez MTA. Role of computed tomography angiography in the diagnosis of vascular stenosis in head and neck microvascular free flap reconstruction. Int J Oral Maxillofac Surg. 2014; 43:811-5. https://doi.org/10.1016/j.ijom.2014.03.014.

20. Delgado Almandoz JE, Romero JM, Pomerantz SR, Lev MH. Computed tomography angiography of the carotid and cerebral circulation. Radiol Clin N Am. 2010;48:265-81. https://doi.org/10.1016/j.rcl.2010.02.007.

21. Saba L. Digital subtraction angiography for the analysis of supraaortic vessels: what is its role nowadays. World J Radiol. 2011;3:147-51. https://doi org/10.4329/wjr.v3.i6.147.

22. Kramer U, Ernemann U, Fenchel M, Seeger A, Laub G, Claussen CD, Miller S Pretreatment evaluation of peripheral vascular malformations using low-dose contrast-enhanced time-resolved 3D MR angiography: initial results in 22 patients. Am J Roent. 2011;196:702-11. https://doi.org/10.2214/AJR.10.5092.

23. Higashihara H, Osuga $K$, Ueguchi T, Onishi H, Tanaka H, Maeda N, Tomoda K, Tomiyama N. Usefulness of contrast-enhanced three-dimensional MR angiography using time-resolved imaging of contrast kinetics applied to description of extracranial arteriovenous malformations: initial experience. Eur J Radiol. 2012:81:1134-9. https://doi.org/10.1016/j.ejrad.2011.03.040.

24. Razek AA, Gaballa G, Megahed AS, Elmogy E. Time resolved imaging of contrast kinetics (TRICKS) MR angiography of arteriovenous malformations of head and neck. Eur J Radiol. 2013;82:1885-91. https://doi.org/10.1016/j.ejrad.2013.07.007.

Ready to submit your research? Choose BMC and benefit from:

- fast, convenient online submission

- thorough peer review by experienced researchers in your field

- rapid publication on acceptance

- support for research data, including large and complex data types

- gold Open Access which fosters wider collaboration and increased citations

- maximum visibility for your research: over $100 \mathrm{M}$ website views per year

At BMC, research is always in progress.

Learn more biomedcentral.com/submission 